\title{
Real time Passenger Flow Calculation Based on Zynq
}

\author{
Yiliang $\mathrm{Wu}^{1, \mathrm{a}}$, Zhansheng yuan ${ }^{1, \mathrm{~b}}$ and Jiachun Zheng $^{1, \mathrm{~b}}$ \\ 1Information Engineering College, Jimei University, Xiamen Fujian 361000, China. \\ a: wuyiliang@jmu.edu.cn
}

Keywords: Passenger; Hough GHT; Xilinx Zynq; Hardware and software collaborative design.

\begin{abstract}
There are many researches on pedestrian detection. These researches are mainly used in ADAS systems based on the front view of the pedestrian. However in some situation, for example on the bus or in the public access, passenger flow calculation needs to be done based on the top view images. This paper presents an efficient FPGA-based passenger flow calculation on a Xilinx Zynq SoC. The algorithms presented include hair model and head detection. The hardware and software collaborative design realized accurate detection in real-time.
\end{abstract}

\section{Introduction}

Passenger flow calculation counts the amount of the people in a certain area through video analysis. The current passenger detection algorithms are mainly based on the front view of the passenger[1]. However in the situation of passenger flow calculation, only the top view images can be captured because of the restricted position of image capture device or to avoid occlusion among the human body as much as possible. In this kind of images, particularly top of the head will be shown unblocked among all of the human body parts. The feathers such as contour of the top of the head and the color distribution in the head region are the only features to distinguish the multiple passengers from complicated circumstance.

There are two categories of approaches presented in literature for passenger flow calculation based on the top view of flow [2]. The first is based on prior knowledge, for example the hair color model or shape etc. The second approach using feather and classifier to detect. The classifier includes SVM、AdaBoost and neural networks. Prior knowledge based approach achieves a model or template to detect human head exactly by gathering lots of experimental data.

Focusing on the real-time detection and counting of human body in top view image, this paper present an embedded solution based on HSI color space and mathematical morphology. In the pretreatment stage prior knowledge of hair color is used to determine the candidate range. The dilation and erosion algorithms are used to get the contour of the target. At last GHT are used to judge the geometrical characteristics of contour are the head or not.

The paper is organized as follows: In Section 1 related work is presented. The algorithm for passenger flow calculation is explained in Section II. The hardware and software implementation are explained in Section III. In Section IV the hardware implementation of passenger flow calculation is compared against the software implementation. Finally, Section V concludes this paper and gives an outlook to future work.

\section{HEAD DETECTION ALGORITHM}

In figure 1, a simplified block diagram of the implemented technique is shown. The system is separated into three major operations. A binarization is applied to input images based on the HSI and hair grey scale[3]. Then the dilation and erosion algorithm are used before edge detection in order to get several candidate areas. At last the GHT (Generalized Hough Transform) is performed on the resulting edge points to judge whether these areas are the head or not. 


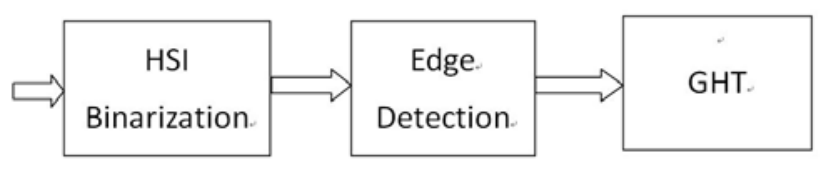

Figure 1. Stage of the detection algorithm.

\subsection{HSI and Its Application in Head Detection}

HSI color space is a model that consistent with the human visual system, which uses three attributes to represent the color value, that is, hue, saturation and intensity. Since the HSI color space separates the illumination intensity and hue into two relatively independent properties, the color space can reduce the illumination change to a certain extent, thus contributing to image segmentation [4]. The common conversion formulas from RGB color space to HSI color space are as follows:

$$
\begin{gathered}
I=R+G+B / 3 \\
H=\frac{1}{360}\left[90-\tan ^{-1} \frac{F}{\sqrt{3}}+(0, G>B ; 180, G<B)\right] \\
F=(2 * R-G-B) /(G-B)
\end{gathered}
$$

Because the HSI color model is more in line with the human visual characteristics, and its $\mathrm{H}$ attribute and I attribute is relatively independent, so consider the use of the color space on the hair color modeling, so as to achieve the hair from the color image for the purpose of segmentation. In this study, a large number of human head samples were taken for experiments and analysis. The experimental results show that the hair color has a good clustering effect in the HSI space, and the clustering interval of the hair color in the HSI space is statistically [5]:

$$
H=[0,10] \cup[110,130] \cup[160,180]
$$

Therefore, in the pre-processing of the image $\mathrm{H}$ value of the interval can be used to find all the head candidate area, and then split and judge. After a large number of experiments, this study found that the gray value of human hair color is generally not greater than 70 , according to the gray value and the value of $\mathrm{H}$ together determine the candidate head area, and can be binarized. The binarization of the original image according to equation (5) is as follows:

$$
f(i, j)=\left\{\begin{array}{c}
0, i f h(i, j) \in(0,10] \cup[110,130] \cup[160,180] \operatorname{andg}(i, j) \leq 70 \\
255, \text { others }
\end{array}\right.
$$

\subsection{Edge Detection}

There are several connected regions in the binarized image, especially in head candidate area. There may be holes or burrs on the edge in these areas. So the dilation and erosion are used on binary images. Canny edge detector extracts the edge points from the previous results.

\subsection{Character judgment}

To extract the head's contour feather in top view image exactly with lower complexity algorithm and avoid false recognition as much as possible, this paper adopts GHT as the main technique to extract the head's quasi-circle contour.

\section{Embedded System Implemention}

Xilinx Zynq-7000 all programmable SoCs combines an ARM dual-core processing system (PS) with programmable logic (PL) [6]. Image acquisition, head detection, and high definition display are accomplished in this system by the combination of hardware and software in the platform of Zynq as shown in Figure 2. 


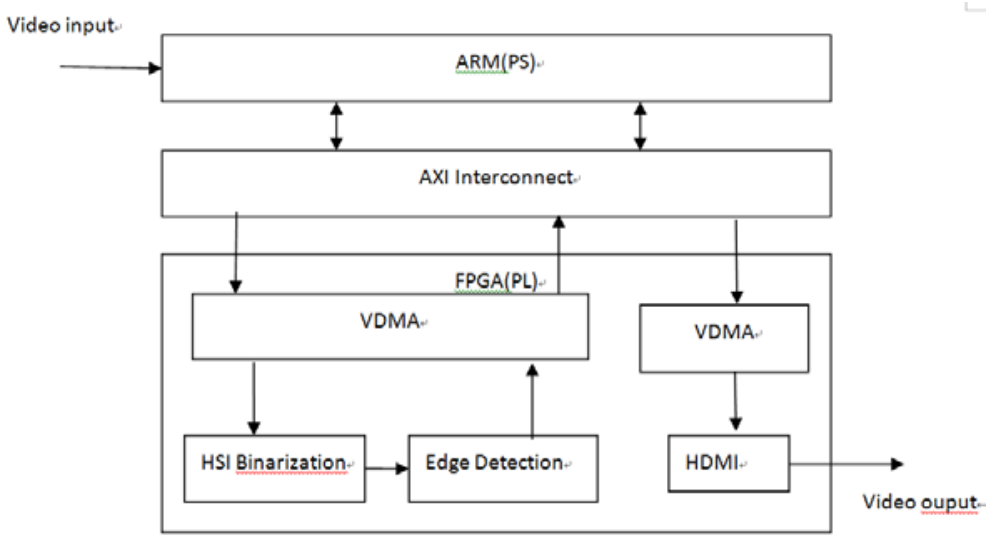

Fig 2. System framework.

Our test board is equipped with a Zynq z7010 SOC. The design is accomplished both in the Processing System(ARM CPU) and in the Programmable Logic(FPGA). Communication between these two components is provided through AXI Protocol. The video is loaded inside the ZYNQ from a camera interface. The results are displayed on a monitor screen through an HDMI interface that is connected to the programmable logic. The Video Direct Memory Access IP (VDMA in Figure 3) provided by Xilinx is used to accelerate the transmission rate.

The hardware-accelerated IP core in this design includes image HSI binarization and grayscale image edge extraction. Through Xilinx's Vivado_HLS tools the design of the IP core can reduce design time. The hardware RTL code is generated by writing the $\mathrm{C} / \mathrm{C}++$ algorithm in the Vivado_HLS tools [7]. Human head features are detected by the software in the PS part of the design.

\section{Experiment}

Our test prototype is the Zedboard, equipped with a Zynq z-7010 SOC from Xilinx.The feasibility and effectiveness of the algorithm was verified. Specific experimental results was shown in Figure 3 .

(a) for the captured original image, (b) for the preprocessed binary value ,(c) is the result of the closed operation for (b), and (d) is the result of Canny edge detection of the image, (e) for the graph closed operation of (d),(f) is a result of the feature evaluation after the contour is extracted,The red circle marks the result image of the head position.

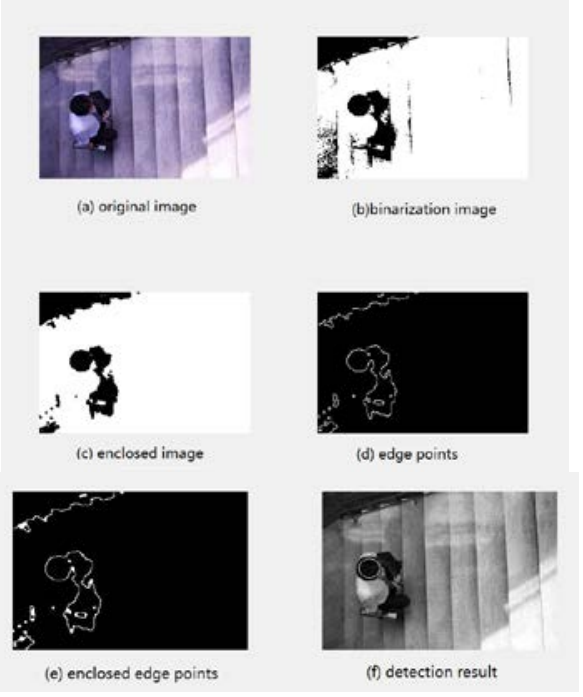

Fig 3. Head detection results.

\section{Conclusions}

In this paper, a real time passenger flow calculation system that combines hardware and software 
co-design is implemented on Xilinx's ZedBoard, which have advantages over other platforms in terms of the speed, power consumption, development difficulty and cost. The system process images with $640 * 480$ pixels at 50 frames per second. The processing speed and accuracy meets the present requirement. Further work is to improve the frame resolution by exploiting NEON cores in the ARM processor.

\section{References}

[1]Dalal, N.; Triggs, B., "Histograms of oriented gradients for human detection," Computer Vision and Pattern Recognition, 2005. CVPR 2005. IEEE Computer Society Conference on, vol.1, no., pp.886,893vol. 1, 25-25 June 2005.

[2]XU Peizhi, XU Guili, HUANG Xin. Head detection based on randomized hough transform [J] . Computer Engineering, 2012, 38( 1) : 199-200.

[3]Y. Wang, Z. Zhou, E. K. Teoh, and B. Su, "Human hair segmentation and length detection for human appearance model,” in Proc. 22nd IEEE Int. Conf. (ICIP), Aug. 2014, pp. 450-454.

[4]N. Wang, H. Ai, and F. Tang, “What are good parts for hair shape modeling?” in Proc. CVPR, Jun. 2012, pp. 662-669.

[5]X.-Y. Wang, T. Wang, and J. Bu, "Color image segmentation using pixel wise support vector machine classification,” Pattern Recognit., vol. 44,no. 4, pp. 777-787, Apr. 2011.

[6]Xilinx Inc. UG585, “Zynq-7000 extensible processing platform technical reference manual”, 2012.

[7]Xilinx Inc. Xapp890, “Zynq all programmable soc sobel filter implementation using the vivadio HLS tool”.2013 